${ }^{1}$ Department of Medical Surgical Nursing, School of Nursing and Midwifery, Tehran University of Medical Sciences, Tehran, Iran

${ }^{2}$ Department of Adults Health Nursing, School of Nursing and Midwifery, Isfahan University of Medical Sciences, Isfahan, Iran

\title{
Unsuccessful diabetes management: a qualitative study
}

\section{ABSTRACT}

Background. Understanding the experiences of diabetes of unsuccessful management will help the treatment team to plan appropriate interventions to solve the problems they face. The aim of this study was to explore the experiences of unsuccessful diabetes management in people with diabetes.

Methods. A hermeneutic phenomenological method was used to gain the experience of subjects. Participants were selected through purposive sampling. The data were collected through in-depth interviews and analyzed based on Dickman's method.

Results. Data analysis revealed the following three categories: missed care, extreme poverty, and the recurrence of diabetes.

Conclusion. Findings indicated the missed care due to laziness in care, inadequate knowledge, negligence in prevention and not giving up the pleasure, and the scary nightmare that was associated with grief and insolvency. This phenomenon is a recurrent manifestation of the disease and may result in incurable illness and cripple. This in-depth understanding demonstrates the importance of patients' emotional and informational support depending on their inadequate knowledge and altered mental status, which should be considered by health care providers in designing

Address for correspondence:

Leila Mardanian Dehkordi

Department of Adults Health Nursing

School of Nursing and Midwifery

Isfahan University of Medical Sciences

Isfahan, Iran

e-mail: mardanian@nm.mui.ac.ir

Clinical Diabetology 2021, 10; 2: 195-199

DOI: 10.5603/DK.a2021.0012

Received: 02.11.2020

Accepted: 29.12 .2020 appropriate programs to improve diabetes management. (Clin Diabetol 2021; 10; 2: 195-199)

Key words: diabetes management, qualitative study, phenomenology

\section{Introduction}

According to the International Diabetes Federation, there were 415 million diabetic people worldwide in 2015, and the number is predicted to increase by 642 million by 2040 [1]. Mismanagement of disease leads to reduced quality of life and disability in the affected population and the risk of death among people with diabetes is twice than of people with other conditions [2]. It also imposes significant economic costs on the individuals, their families, and the community. Evidence shows that direct and indirect economic cost of diabetes was $\$ 327$ billion in 2012, and increased by $26 \%$ from 2012 to 2017 [3].

Adherence to diabetes treatment regimen and self-management remains the most important the determinant of achieving clinical goals (ie, blood glucose control) [4-5]. However, many unknown barriers prevent adherence to the medical treatment regimen and self-management, which reduce the level of health and quality of life in individuals [6]. In spite of health care providers' interventions, evidence indicates that management of the disease is poor and prevalence of complication of unsuccessful diabetes management is high $[7,8]$. Results of a study by Van Stone et al. (2015) shows, uncontrolled diabetes leads to psychological distress, negative mood, cognitive problems, irritable and aggressive behavior, problems in establishing relationships and self-image, and decreased self-esteem [9].

Since diabetes is a chronic and progressive disease, the management of the disease is very important to prevent worsen outcomes. The studies have been shown 
that the disease management programs specialty in Iran were not designed by the perspective of patients, their needs, preferences, and abilities. Therefore by understanding the unsuccessful diabetes management experience of patients, we can conclude the elements that can be affected the ability of patients to diabetes management and consider them when following the patients. But there is little information about the experience of unsuccessful diabetes management, so this study aimed to explain the experiences of people with diabetes from unsuccessful disease management in the hope that identifying their experiences would help health planners to design appropriate interventions for these people and provide strategies to improve the health and quality of life of patients and reduce the individual, family and social impacts of this phenomenon.

\section{Methods}

Using the interpretative phenomenological approach, we conducted this qualitative study to demonstrate and explain the unsuccessful management of diabetes experienced by people with diabetes. In this study, participants were selected using a purposeful sampling method. They included people with diabetes who were unsuccessful in diabetes management and refer to the health care center to control glycemia or cure complications of uncontrolled diabetes.

Before every interview, the researcher introduced herself and explained the main aims of the study and then data were collected through individual face-toface, in-depth, and semi-structured interviews and were continued until no new ideas emerged. Additionally, 10 people who experienced unsuccessful management of diabetes have taken part in this study.

Every interview started with open questions such as "would you please tell me about your experience of uncontrolled diabetes? What does the mismanagement of diabetes mean to you?" The participants were free to express their feeling and experience. Then, according to the answers provided by the participants, more detailed questions were asked to reach the result and goal of interviews such as "can you explain more? What is the meaning of the sentence you told? How do you feel about this issue? The location of the interview was determined according to the preference of the participants. Length time of interview varied between 40 and $60 \mathrm{~min}$ and with an average of $45 \mathrm{~min}$ and were recorded with the permission of the interview.

Each interview was listened several times then the audio recording was transcribed into texts in MSWord. Dickelman et al.'s seven-step analysis was used to analyze qualitative data. In stage one transcriptions were read several times to get a general idea of the interview. In stage two, interpretative abstract for each interview was written by the researcher and the underlying themes emerged extracted themes inserted into related categories. In stage three, research team members discussed the extracted themes again and shared their ideas in this regard and discussed disagreements to reach consensus emerged themes. Later interviews completed the previous themes obtained during earlier analysis, and sometimes, lead to new emerged themes; interpretations and patterns were developed while finalizing themes. In stage four frequent shifting back and forth to the transcriptions for clarifying, and new classifying of extracted themes and resolving any inconsistencies among research team members' and / or interpretations were performed. In stage five finding recurrent themes in the texts which conveyed shared experiences in participants about unsuccessful diabetes management was done. In stage six the transcriptions were compared, and the themes were discussed in terms of their relationship with one another. In stage seven, a "constitutive pattern" was formulated, and its final version was delivered to the interpretation group to be examined [10].

For determining the accuracy of data, Lincon and Gaba evaluation scale was used [11]. Therefore the study validity was ensured by selecting the appropriate context, resources, and eligible participants, close, long-term, and continuous interaction with the participants, adopting a team approach using collective team discussion for reaching subthemes and themes, analytical comparisons and frequent and reciprocal referral to raw data, identifying and clarifying process steps to facilitate their understanding by others, providing a draft of themes and phrases selected from the texts to team members and experts to extract and apply their comments and suggestions in writing of the final version of the plan report.

\section{Findings}

In total, 10 people with the most variation in gender ( 5 female and 5 male) and marital status ( 7 married and 3 single persons) with age range of $28-80$ years and 7-36 years history of diabetes participated in this study.

In the process of data analysis 3 main themes and 8 sub-themes emerged including missed care (laziness in care, inadequate knowledge, and not giving up the pleasure), extreme poverty (grief, insolvency, scary nightmares) and recurrence of diabetes (being a cripple and incurable ill).

\section{Missed care}

This theme with three sub-themes of "laziness in care", "inadequate knowledge", and " not giving up 
the pleasure " was the main cause of unsuccessful disease management, which leads to the loss of needed care to control the symptoms and complications of the disease.

\subsection{Laziness in care}

Laziness in care refers to low-quality care and negligence in treatment follow up. One participant in this regard stated: "It's all laziness... negligence and laziness go hand in hand to reach this stage. It was our own inattention that made it worse. It was easy to control it earlier, at least this problem could be prevented..." - p 1.

Another one said: "One has to be careful from the beginning. Hey, don't be like me. You must be very serious... serious".

\subsection{Inadequate knowledge}

Inadequate knowledge represents the lack of essential knowledge for successful disease management. A participant in this regard stated: "I went to the physician and he said that your blood glucose is 400 , which is high. I didn't understand the means of this sentence and didn't know what to do" - p 3.

Another contributor said: "I didn't know much about it. I thought this rise in blood glucose and side effects of the disease was normal events when blood glucose level rises and is not treated with pills. I thought it would happen for everyone with diabetes and I can do nothing about it" - p 5.

\section{Not giving up the pleasure}

Inattentiveness and involvement by enjoyment was some of the factors that contributed to the mismanagement of the disease. One participant in this regard stated: "Well, I love sweets. Yesterday they were eating sweets in front of me, so I couldn't stop myself and had some too" - p 4.

Another participant said: "After all, eating s is enjoyable, eating sweets a pleasure in life. Some become addicted to drinks cola and fast-food... Eating these foods is a joy... I don't pay attention to don't eating recommendations. I go my own way and eat whatever I like" - p 5.

\subsection{Extreme poverty}

Extreme poverty is a learned form of insolvency that is associated with a sense of grief and a nightmare. In this situation, the individual is reluctant to control the disease and feels he has been left in the abyss.

\subsection{Grief}

The experience of unsuccessful management was an expression of perceived grief, which is associated with emotions such as anger, regret, and disappointment.
One participant expressed her anger by complaining from God: "I'm not feeling well. I'm so tired and say to myself why me? Why I have so much trouble in this world, my problems have accumulated" - $p 8$.

Another participant felt sorry: "We could have stopped it but we didn't. Now, we can do nothing except for regret" - p 1.

The discontinuation of hope for treatment management and disease control was echoed in the participants' words: "I get upset about why it can't be controlled. My legs hurt and I am not very active. I have also no control of blood glucose levels. I feel hopeless when I take insulin and my blood glucose level is not controlled" - p 9 .

\subsection{Insolvency}

The inability to manage diabetes had made participants feel helpless. One participant in regard to the feeling of inability to control the disease said: "I can't do anything else... What can I do?... What should I do? I don't know what I can do... My blood glucose is high and there is nobody here me. I get up and take a pill (Glibenclamide) to see what happens. This is my life" - p 4.

Another participant spoke of perceived incompetence that results from her inability to control the disease: "Well, for me it means that I can't control my blood sugar well and I feel inefficiency and inability to control my illness".

\subsection{Scary nightmare}

Unsuccessful disease management was like a scary nightmare for participants that resembles death and loneliness. "For me, it's like continuous unrest and pain. A restlessness and constant fear and nightmare that is with you in all time. It is like an unknown future and facing a bad thing that you always expect to happen. It is heavy psychological stress" - p 10 .

Another participant stated: "Always bad memories are recalled for me. My 23-year-old sister didn't know she had diabetes. She went to the hospital and received glucose. She went to a coma and died. My brother had high blood glucose and was ignoring it until he died at the age of 55. I lost all my family, my father, and mother" - P 7 .

\section{Recurrence of diabetes}

Finally, unsuccessful management of the disease manifested itself as recurrence of the disease, which meant incurable illness and becoming a cripple.

\subsection{Becoming cripple}

Participants saw unsuccessful management as a crippling and limiting phenomenon, a reminiscent of 
hospitalization. A participant described the negative effects of unsuccessful management on daily life: "If you can't control your disease, your family and friends would get hurt. I did not want to involve my family, but it affects your relationship whether you like it or not. You cannot work and you would be imprisoned in the same situation. You always think about high and low levels of blood glucose as if you are not living at all" - p 10.

A participant talked about his previous hospitalization: "I remember being in the hospital... With patients whose faces were depressed or their legs became black due to diabetes... I think of a funeral home that is a similar place... It hurts" - p 5.

\subsection{Incurable illness}

The participants referred to unsuccessful disease management as an incurable illness. An incurable phenomenon that is a particular form of the disease exacerbation and causes diabetes complications: "I'm just upset and scared that it leaves irreparable damage to the rest of my body and massively affect my work and life. I am afraid of early death and all other complications that may arise. If I had started ten years ago, I was much better off now. Now it's too late. It has no cure. Even if it has a cure, it would be very difficult to stop it" - p 1.

"Increasing my dosage makes me worried that I will not respond to medication anymore. I worry that my blood sugar level may stay up all the time and causes me to be hospitalized and kill me" - p 10 .

\section{Discussion}

One of the prominent themes found in this study was missed care, which included subscales of inadequate knowledge, negligence in prevention and follow-up, laziness in care, and not giving up on pleasure. Other studies have also referred to individuals' attitudes, beliefs, knowledge, financial constraints, and social support as barriers to diabetes management [12]. The findings of Jam (2018) are in line with the results of present study show that patient education affects their attitudes and adherence to treatment regimens and subsequently, successful disease management [13]. Mousavi Zadeh et al. (2016) refer to lack of training and counseling as a barrier of management, neglected inevitable necessity, that due to poor performance of the treatment team [14]. Mardanian and Abdoli (2017) state that training provided to people with diabetes ranges from useful to repetitive, and suggest that in order to be effective, training should be trained based on individual's needs and should be carried out by expert instructors [15].
Other findings of the present study were negligence in follow-up and laziness in care, however, diabetes belongs to the individual and affects individuals and diabetics are in control of their disease and decisions so they experience the consequences of their daily decisions, and health care providers are solely responsible for the quality of care and the education they provide [15]. Effective self-management of diabetes requires the psychological and social support that should be provided for patients [16].

Unsuccessful disease management in this study was associated with a feeling of grief that comes from lack of control, and the participants were experiencing feelings of disbelief, anger, frustration, and regret. In Starkman's study, diabetic people were also experiencing similar feelings including frustration, guilt, anxiety, and anger over uncontrolled diabetes [17].

In this study, unsuccessful management was like a frightening nightmare for the participants that resembled death and loneliness. In Balf's (2013) study, the common sense of fear of death among participants was reported too [18].

Insolvency was another emotion experienced by participants who failed to manage their disease. In line with this finding, Mousavizadeh refers to personal insolvency as one of the barriers to successful disease management [14].

The reccurrence of diabetes was another finding of this study, which resembled the sense of limitation and paralysis for the participants; an experience that patients understood and referred to with the phrases such as always ill, disabled and deprived of normal living [19]. Fear of side effects seen in this the study can also be seen in Balf's study [18].

\section{Conclusions}

Patients' experiences of unsuccessful disease management indicated missed care that results from laziness in care, inadequate knowledge, negligence in follow-up, and not giving up the pleasure. Unsuccessful disease management makes the patient frustrated and is a frightening nightmare for them, which is associated with grief and insolvency. The notion that a person is able to exclude himself from complications can suddenly change. This phenomenon is a recurrent manifestation of the disease and may lead to incurable illness and pain; a state where medical interventions have no effect whatsoever. Experience of the participants demonstrated the importance of emotional and informational support for patients with inadequate knowledge and altered mental status, which should be considered by policymakers in designing appropriate programs to improve diabetes management. 


\section{Research involving human participants, their data or biological material}

The Ethics Committee of Tehran University of Medical Sciences has approved this study with ethical code IR.TUMS.VCR.REC.1398.579.

The aims of the study explained for participants and they were also reassured that their participation was voluntary and they could withdraw from the study at any time.

Permissions were taken for recording the interviews from them. They were assured of anonymity and confidentiality of their identity.

\section{Acknowledgments}

The authors would like to thank the participants for their cooperation in this study, Professor Bodil Rasmussen for her comments on this manuscript, And Tehran University of science for its financial support.

\section{Conflict of interest}

The authors declare no conflict of interest.

\section{REFERENCES}

1. International Diabetes Federation. IDF Diabetes Atlas, 7th ed. Brussels: International Diabetes Federation, 2015.

2. World Health Organization. Global Report on Diabetes. Available online: http://apps.who.int/iris/bitstre am/10665/204871/1/9789241565257_eng.pdf. [Last accessed: 10.8.2017].

3. American Diabetes Association. Economic costs of diabetes in the U.S. in 2017. Diabetes Care. 2018; 41: 917-928.

4. Davies MJ, Gagliardino JJ, Gray $\sqcup$, et al. Real-world factors affecting adherence to insulin therapy in patients with type 1 or type 2 diabetes mellitus: a systematic review. Diabet Med. 2013; 30(5): 512-524

5. Riaz I, Riddle MC, Rosenstock J, et al. Personalized management of hyperglycemia in type 2 diabetes: reflections from a Diabetes Care Editors' Expert Forum. Diabetes Care. 2013; 36(6): 1779-1788.

6. Munshi MN, Segal AR, Suhl E, et al. Assessment of barriers to improve diabetes management in older adults: a randomized controlled study. Diabetes Care. 2013; 36(3): 543-549, doi: 10.2337/dc12-1303, indexed in Pubmed: 23193208.
7. Undavalli V, M. P, H. N. Prevalence of undiagnosed hypertension: a public health challenge. International Journal Of Community Medicine And Public Health. 2018; 5(4): 1366, doi: 10.18203/2394-6040.ijcmph20180974.

8. ALSULIMAN, Mohammed, et al. Prevalence and Risk Factors for Poor Glycemic Control among Patients with T2DM in Saudi Arabia: A Systematic Review and Meta-Analysis of Prevalence. 2019.

9. Vanstone M, Rewegan A, Brundisini F, et al. Patient perspectives on quality of life with uncontrolled type 1 diabetes mellitus: a systematic review and qualitative meta-synthesis. Ont Health Technol Assess Ser. 2015; 15(17): 1-29, indexed in Pubmed: 26649106.

10. Allen D, Tanner CA. Diekelmann N, Allen D, Tanner CA. Hermeneutic analysis of the NLN criteria for the appraisal of baccalaureate programs. The NLN criteria for appraisal of baccalaureate programs: a critical hermeneutic analysis. National League for Nursing: New York 1989; 11-34. ; 19.

11. Speziale, HS \& Carpenter DR. Qualitative research in nursing: advancing the humanistic imperative, Philadelphia, Wolters Kluwer Health/Lippincott Williams \& Wilkins 2011.

12. Nam S, Chesla C, Stotts NA, et al. Barriers to diabetes management: patient and provider factors. Diabetes Res Clin Pract. 2011; 93(1): 1-9, doi: 10.1016/j.diabres.2011.02.002, indexed in Pubmed: 21382643.

13. Jaam M, Hadi MA, Kheir N, et al. A qualitative exploration of barriers to medication adherence among patients with uncontrolled diabetes in Qatar: integrating perspectives of patients and health care providers. Patient Prefer Adherence. 2018; 12: 2205-2216, doi: 10.2147/PPA.S174652, indexed in Pubmed: 30410316.

14. Mousavizadeh SN, Ashktorab T, Ahmadi F, et al. Evaluation of barriers to adherence to therapy in patients with diabetes. J Diabetes Nurs. 2016; 4(3): 94-108.

15. Mardanian Dehkordi L, Abdoli S. Diabetes self-management education; experience of people with diabetes. J Caring Sci. 2017; 6(2): 111-118, doi: 10.15171/jcs.2017.011, indexed in Pubmed: 28680865.

16. Chew BH, Shariff-Ghazali S, Fernandez A. Psychological aspects of diabetes care: Effecting behavioral change in patients. World J Diabetes. 2014; 5(6): 796-808, doi: 10.4239/wjd.v5.i6.796, indexed in Pubmed: 25512782.

17. STARKMAN, Harold, et al. Listening to adolescents with uncontrolled diabetes, their parents and medical team. Families, Systems, \& Health, 2019, 37.1: 30.

18. Balfe M, Doyle F, Smith D, et al. What's distressing about having type 1 diabetes? A qualitative study of young adults' perspectives. BMC Endocr Disord. 2013; 13: 25, doi: 10.1186/1472-6823-13-25, indexed in Pubmed: 23885644.

19. Abdoli S, Abazari P, Mardanian L. Exploring diabetes type 1-related stigma. Iranian journal of nursing and midwifery research, 2013, 18.1: 65-70 